Nikolai Ivanovich Vavilov, travelling the world in the early twentieth century to amass a vast collection of plant seeds, was an early explorer of the long tail of plants.

From the perspective of scholarly communication, librarians, publishers and academic institutions have much to gain from the ideas in this book. Scientific content, such as data sets and peer-reviewed papers, is being produced at a breathtaking pace, yet there are still gaps in terms of availability and discoverability. The techniques used to locate previously hard-to-obtain music tracks, obscure books and unexpected content relationships can be adapted to enable researchers to discover lesscited but potentially useful data and papers. Richard Akerman is at the Canada Institute for Scientific and Technical Information, Ottawa, Ontario K1A 0R6, Canada.

Supplementary links for this review are available at www.connotea.org/user/scilib/tag/longtailreview

\title{
Sensitive to modern life
}

\section{Allergy: The History of a Modern Malady \\ by Mark Jackson \\ Reaktion: 2006. 256 pp. $€ 25, \$ 39.95$}

\section{Peter J. Barnes}

The term 'allergy' was first used by the Austrian paediatrician Clemens von Pirquet in 1906. He was describing an exaggerated biological reactivity to foreign substances, which he demonstrated by means of injections. Asthma, eczema and hay fever had been recognized since antiquity, but in the early 1900 s these allergic diseases were considered uncommon. Their increase in prevalence in the past century is unlikely to be due to better diagnosis, as they are usually easy to recognize. In the past 20 years in particular there has been a dramatic increase in allergic diseases of all types in industrialized countries. This is much too rapid to be accounted for by genetic changes, although we know that genetic predisposition is important in the development of allergies, so there must be an environmental cause. The increase in allergic diseases seems to relate most closely to the adoption of a Western lifestyle.

In Allergy, Mark Jackson, professor of the history of medicine at the University of Exeter, UK, traces the historical development of allergy, discusses possible explanations for the recent explosive increase, and concludes that it is a disease of civilization. The book provides a perceptive insight into the historical development of allergy, indicating how thinking changes. It gives fascinating vignettes of key researchers involved in the history of allergy and contains some interesting anecdotes about their lives.

Various environmental causes have been suggested for the increase in allergic diseases, including changes in diet, poorly ventilated housing, increased exposure to allergens, air pollution (commonly believed by patients to be important) and, most convincingly, reduced infections during early childhood, which favours an allergic pattern of immunity. No single factor accounts for the rise, however, which is likely to be caused by a combination of environmental factors that occur together in Western society. Asthma, allergic rhinitis (including hay fever) and eczema have all increased, suggesting that they have an underlying allergic mechanism. Many epidemiological studies have now been done, but it is still not known exactly which environmental

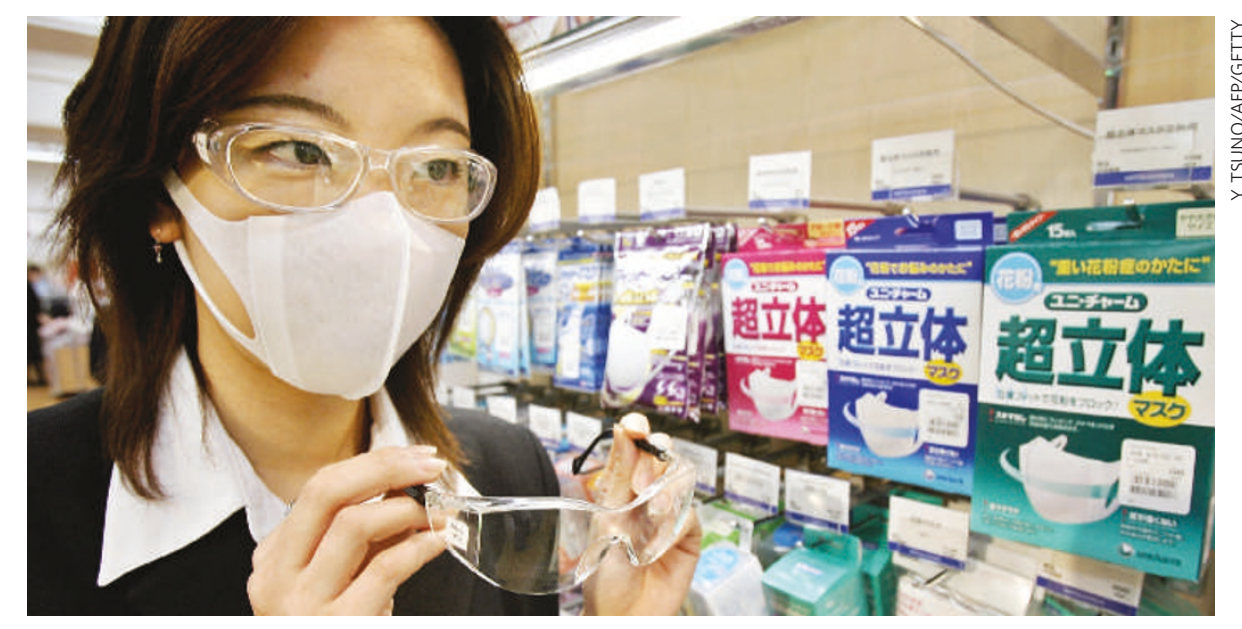

Masks and goggles are the order of the day as Japan struggles to cope with a rise in hay fever.

factors are important in the development of allergies in genetically predisposed patients.

The most compelling evidence for an environmental cause is the high prevalence of allergies in the former West Germany compared with a very low prevalence in the East. These differences are now disappearing in the united Germany as the environmental conditions become more similar. Other convincing evidence is the low prevalence of allergic diseases in children brought up on animal farms, where there is a high level of exposure to endotoxins, which stimulate a protective pattern of immunity. The lack of infections and endotoxin exposure associated with improved hygiene and the widespread use of antibiotics fails to stimulate this protective immunity. Jackson discusses these theories, although he does not provide much evidence for and against each idea.

Allergies are costly, in terms of medication, hospital admissions from asthma, and time taken off work. It is estimated that allergic diseases cost more than $£ 1$ billion (US $\$ 1.9$ billion) in Britain alone, excluding hospital costs. The good news is that the rise in allergic diseases seem to be slowing down. In some countries, $40-50 \%$ of allergy skin tests are positive, and this may be as high as the genetic predisposition will allow. Allergic diseases have been very profitable for pharmaceutical companies, and asthma medications are one of the fastest-growing markets in the world. But the high costs of medication have promoted a search for non-pharmacological approaches centred on changing the environment. Allergen avoidance has usually been disappointing, as it has been impossible to completely avoid exposure, and changes in diet (more antioxidants and increased consumption of oily fish) have not been effective.

Perhaps the most promising approach to allergic disease is vaccination, which is particularly effective against hay fever and in desensitizing against insect stings. Desensitization - the administration of gradually increasing doses of an allergen to promote a long-term change in the immune response - is discussed at various points in the book. However, the treatment can sometime provoke exaggerated responses or even anaphylactic shock, prompting the search for safer approaches. Treatments placed under the tongue, rather than given as skin injections, are effective, and purified allergen proteins may be safer. In the future, DNA vaccines and T-cell peptides could provide a means of inhibiting the allergic response.

Jackson's succinct and clearly written book is aimed at the informed lay reader. $\mathrm{He}$ admirably avoids using jargon and scientific terminology, and gives fascinating insight into the rise in allergic diseases and how this is linked to our modern lifestyle. I recommend this book, which helps us to understand the relationship between health and the environment, and explains why modern living can be detrimental to our health.

Peter J. Barnes is head of respiratory medicine,

National Heart and Lung Institute, Imperial

College London, London SW3 6LY, UK. 